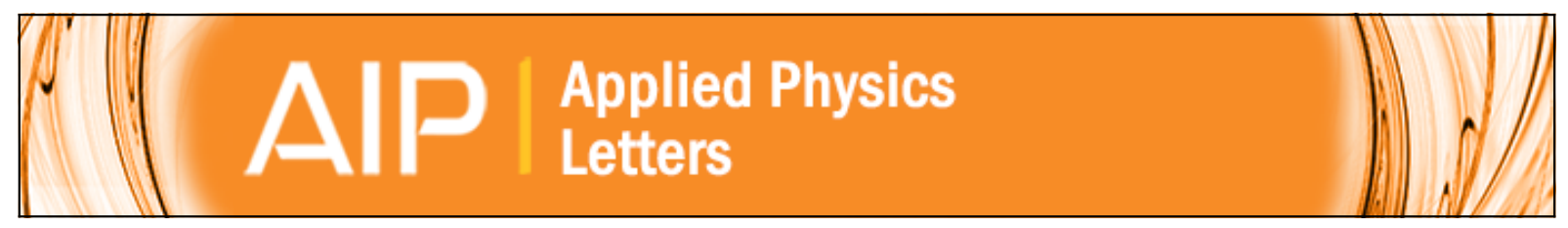

\title{
Bidirectional resonant tunneling spin pump
}

David Z.-Y. Ting and Xavier Cartoixà

Citation: Applied Physics Letters 83, 1391 (2003); doi: 10.1063/1.1602158

View online: $\mathrm{http}: / / \mathrm{dx}$.doi.org/10.1063/1.1602158

View Table of Contents: http://scitation.aip.org/content/aip/journal/apl/83/7?ver=pdfcov

Published by the AIP Publishing

\section{AlP}

\section{Create a profile. \\ Sign up today!}




\title{
Bidirectional resonant tunneling spin pump
}

\author{
David Z.-Y. Ting ${ }^{\text {a) }}$ \\ Jet Propulsion Laboratory, California Institute of Technology, Pasadena, California 91109
}

Xavier Cartoixàb)

T. J. Watson, Sr., Laboratories of Applied Physics, California Institute of Technology, Pasadena, California 91125

(Received 6 March 2003; accepted 27 June 2003)

\begin{abstract}
We propose a mechanism for achieving bidirectional spin pumping in conventional nonmagnetic semiconductor resonant tunneling heterostructures under zero magnetic field. The device is designed specifically to take advantage of the special spin configuration described by the Rashba effect in asymmetric quantum wells. It induces the simultaneous flow of oppositely spin-polarized current components in opposite directions through spin-dependent resonant tunneling, and can thus generate significant levels of spin current with very little net electrical current across the tunnel structure, a condition characterized by a greater-than-unity current spin polarization. We also present modeling results on temperature dependence and finite device size effects. (C) 2003 American Institute of
\end{abstract} Physics. [DOI: 10.1063/1.1602158]

Concepts for creating spin polarized current sources using nonmagnetic semiconductor resonant tunneling heterostructures reported to date include the intraband asymmetric resonant tunneling structures, ${ }^{1}$ the triple-barrier resonant tunneling diode (TB-RTD), ${ }^{2}$ and the asymmetric resonant interband tunneling diode (aRITD). ${ }^{3}$ All of these structures contain asymmetric quantum wells where quantized states are spin split by the Rashba effect, ${ }^{4}$ which describes the lifting of spin degeneracy due to structural inversion asymmetry. Spin filtering is accomplished by exploiting the fact that the spin of a resonantly transmitted electron aligns with that of the quasibound state traversed. ${ }^{5,6}$ Quantum well quasibound state spin directions are perpendicular to the growth direction $\left(z\right.$ axis) and to the in-plane wave vector $\mathbf{k}_{\|} \cdot{ }^{7}$ Figure 1 shows that when the spin directions of two spin-split subbands are plotted along constant $k_{\|}$contours in the $\mathbf{k}_{\|}$plane, they appear as counter-clockwise (CCW) and clockwise (CW) pinwheels. It illustrates two of the major challenges to achieving efficient spin filtering: (1) $\mathrm{CCW}$ and $\mathrm{CW}$ subband states at the same $\mathbf{k}_{\|}$have opposite spins, and, (2) $+\mathbf{k}_{\|}$and $-\mathbf{k}_{\|}$states within a given spin-split subband have opposite spins. Either property alone could prevent significant net spin polarization in an ensemble of resonantly transmitted electrons originating from a thermal reservoir. Both the TB-RTD and the aRITD have multilayer quantum well designs that can provide strong selectivity between resonant tunneling through the $\mathrm{CCW}$ and $\mathrm{CW}$ subbands, but they also require mechanisms such as lateral $E$ fields in the source region ${ }^{1}$ or onesided collection, ${ }^{2}$ for creating lateral momentum distribution anisotropy to achieve net spin polarization. In this work, instead of treating the pinwheel spin configuration as a difficulty to overcome for spin filtering, we propose a mechanism that exploits this special property to achieve spin separation.

Figure 2 schematically illustrates the basic concept using

\footnotetext{
${ }^{a)}$ Electronic mail: david.z.ting @jpl.nasa.gov

b) Present address: Dept. of Physics, U. Illinois at Urbana-Champaign, Urbana, IL 61801.
}

an aRITD. The device structure consists of an asymmetric composite InAs-GaSb well, surrounded by AlSb barriers and high mobility InAs emitter and collector channels. Electrons can tunnel between InAs electrodes via the GaSb valence subband states. The design exploits large valence band spin-orbit interaction to provide strong spin selectivity, without suffering from fast hole spin relaxation. ${ }^{3}$ We do not intentionally bias the spin pump along the growth $(z)$ direction. Instead we apply a small lateral $E$ field in the emitter region only. The application of an in-plane $E$ field displaces the emitter Fermi surface. As depicted in Fig. 2, the displacement creates an excess of carriers on the $+k_{x}$ side, which can tunnel to the collector, and a deficit of carriers on the $-k_{x}$ side, which becomes available to receive electrons tunneling from the collector. The aRITD structure shown is designed such that resonant tunneling through the $\mathrm{CW}$ states dominates over the CCW states. ${ }^{3}$ Considering the $\mathrm{CW}$ subband only, resonantly transmitted electrons on the $+k_{x}$ and $-k_{x}$ sides will be spin polarized along the $-y$ and $+y$ directions, respectively. The net result is a forward (emitter to collector) electron current with $-y$ spin polarization, and a backward current with $+y$ spin polarization. Note that structures such as the spin-blockade device can also be used in the spin pump; the key requirement is that tunneling through one
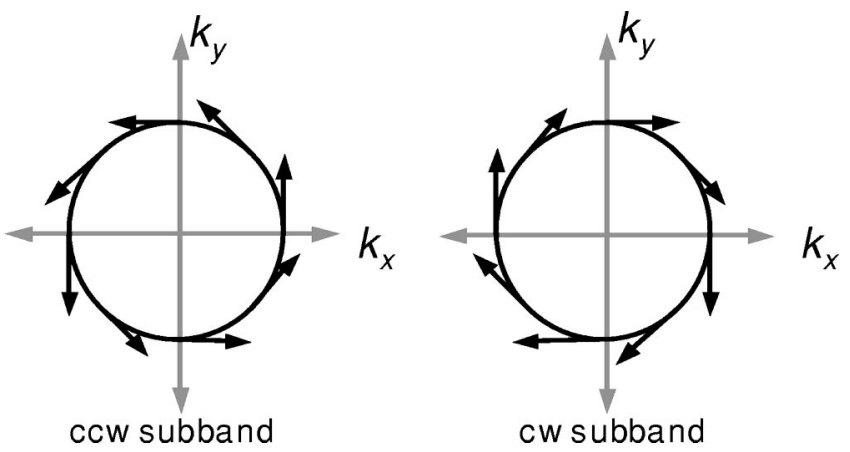

FIG. 1. Spin directions of spin-split subbands in a Rashba effect quantum well along a constant $k_{\|}$contour. 

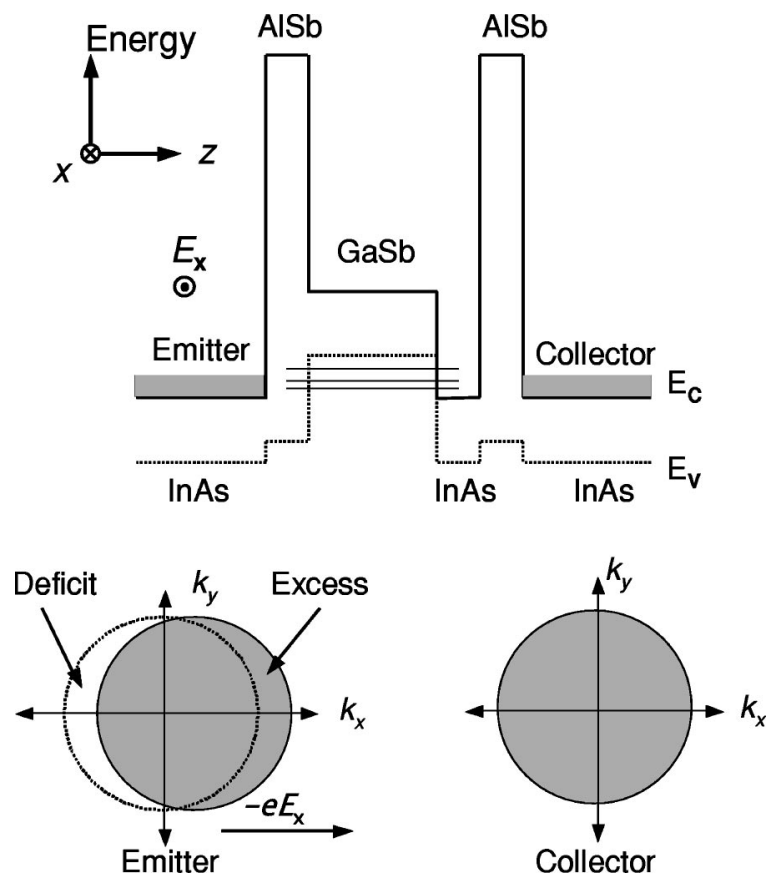

FIG. 2. Schematic energy band diagram of an asymmetric resonant interband tunneling structure used for the bidirectional spin pump. The bottom illustrates the emitter and collector carrier populations in momentum space.

of the spin-split subbands should be much stronger than the other.

Figure 3 shows the spin-dependent current densities and current spin polarization as functions of the lateral $E$ field for a resonant tunneling spin pump, computed using the multiband quantum transmitting boundary method. ${ }^{8}$ We show results for electrode carrier densities selected to reach heavyhole 2 (hh2) light-hole 1 (lh1) and hh1 resonant tunneling

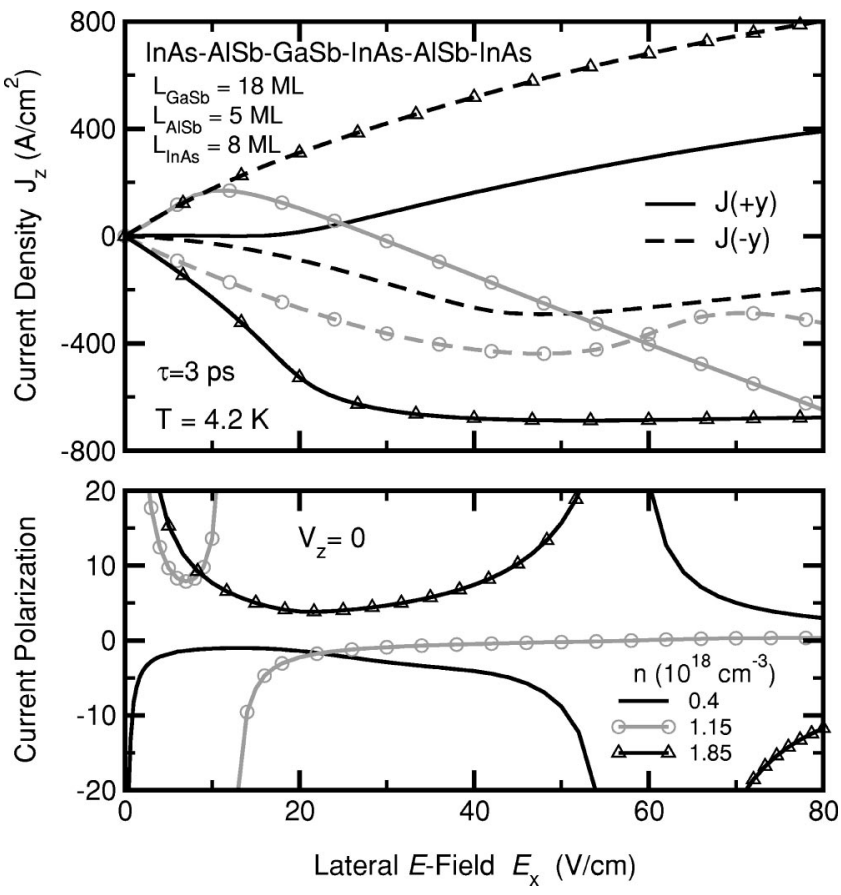

FIG. 3. Spin polarized current density components and current spin polarization as functions of lateral $E$ field for the bidirectional spin pump. Electrode carrier densities of $0.4,1.15$ (circles), and 1.85 (triangles), in units of $10^{18} \mathrm{~cm}^{-3}$ are used to achieve $\mathrm{hh} 2 \mathrm{~h} 1$, and hh 1 resonant tunneling conditions, respectively.

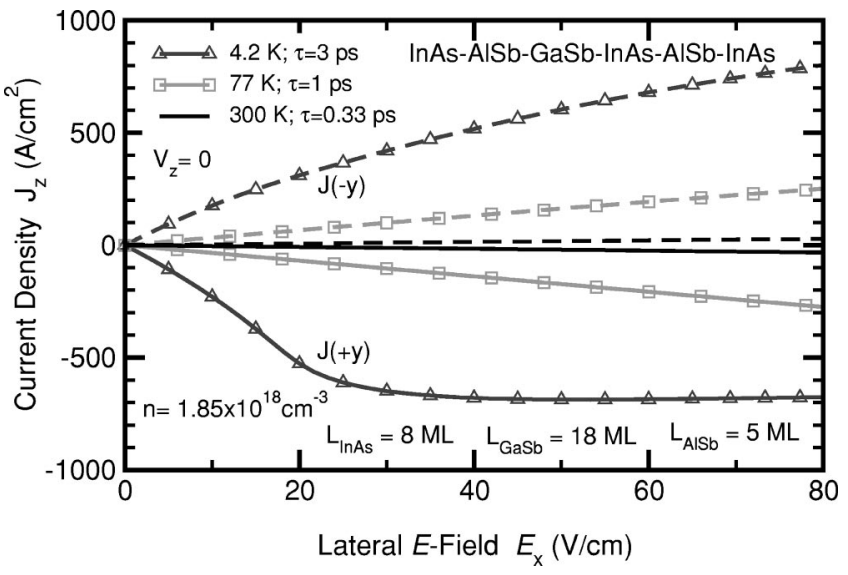

FIG. 4. Spin polarized current density components as functions of lateral $E$ field at different temperatures.

conditions. The hh1 case yields the most favorable result, showing substantial spin up and spin down (defined with respect to the $y$ axis) current flows in opposite directions for modest values of lateral $E$ field. Tunneling via $1 \mathrm{~h} 1$ is less effective because the dominant contribution to $\mathrm{lh} 1$ current comes from small $\mathbf{k}_{\|}$resonant tunneling, which are not spinselective since Rashba spin splitting vanishes at the zone center. The fact that $\mathrm{lh} 1$ could show even limited (at small $E_{x}$ ) bidirectional spin pumping at all is due to a salient feature of the spin pump design: zone center states are occupied in both the emitter and the collector (see Fig. 2), so the spin pump automatically blocks tunneling through zone-center states.

Since the spin polarized current density components $J(+y)$ and $J(-y)$ have opposite signs in a bidirectional spin pump, $P_{J}=[J(+y)-J(-y)] /[J(+y)+J(-y)]$, the current spin polarization, can attain values greater than 1 . In fact, Fig. 3 shows that $\left|P_{J}\right|$ can be quite large when $J(+y)$ and $J(-y)$ are nearly equal in magnitude but opposite in direction. Physically, this means that we could obtain a sizeable spin current with very little net electrical current across the tunnel structure (There is, of course, still $E_{x}$-induced driving electrical current along the $x$-direction).

Figure 4 shows that the effectiveness of the bidirectional spin pump decreases with higher temperature. This is only partially due to carrier thermalization. The main reason for the performance degradation is because momentum relaxation time $\tau$ shortens with temperature increase. The relaxation times used in our calculations are consistent with (and do not exceed) the experimental values for high mobility samples reported in the literature. ${ }^{9}$ In the relaxation time approximation the amount that the Fermi sphere shifts in $k$ space is given by $\Delta k_{x}=-e E_{x} \tau / \hbar{ }^{10}$ As $\tau$ decreases with temperature increase, $\Delta k_{x}$ decreases proportionally. It is easy to see in Fig. 2 that a smaller shift renders the spin pump less effective. This result underscores the importance of using a high mobility emitter channel in the spin pump.

We could compensate for the effect of decrease in $\tau$ by applying a larger lateral $E$ field, but only to a limited extent due to finite-size effects. In the spin pump we apply a small lateral $E$ field in the emitter, but not in the collector. There is no intentional vertical biasing $\left(V_{7}\right)$ across the heterostructure. But in a finite-size device with lateral dimension $W$, 


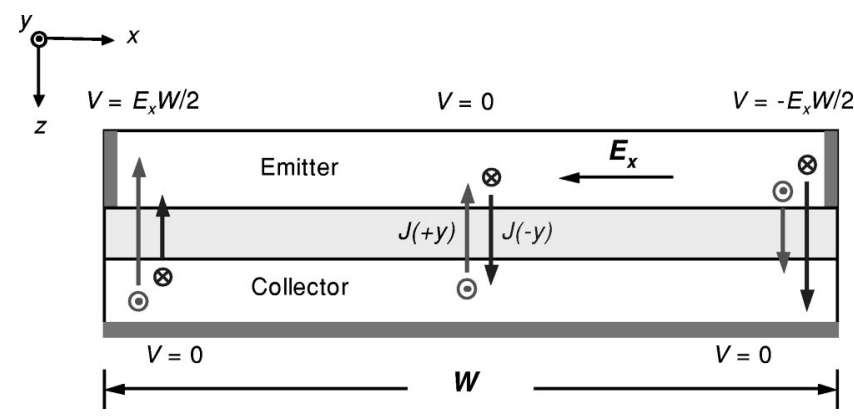

FIG. 5. Schematic illustration of a simplified model used to treat finite-size effect. The vertical arrows indicate the directions of spin-dependent current components.

illustrated in Fig. 5, the presence of a lateral $E$ field in the emitter implies that there is a lateral voltage difference of $\Delta V_{x}=E_{x} W$ between the two edges of the emitter. On the other hand, the absence of lateral $E$ field in the collector implies that there is no lateral voltage variation in the collector. Then as we move laterally from one edge of the device to the other, the vertical bias $V_{z}$ cannot always remain zero; in fact it changes by $E_{x} W$ from edge to edge. To treat this effect, we should in principle use a two-dimensional model. Here we use a simplified model to give us a qualitative picture of the finite-size effect. We model the finite device as a set of parallel diodes under different vertical bias $V_{z}$, ranging from $-E_{x} W / 2$ to $+E_{x} W / 2$. We average device properties over this set of parallel diodes to estimate finite-size effects.

The top panel of Fig. 6 shows the current-voltage characteristics for a single aRITD, with three different values of
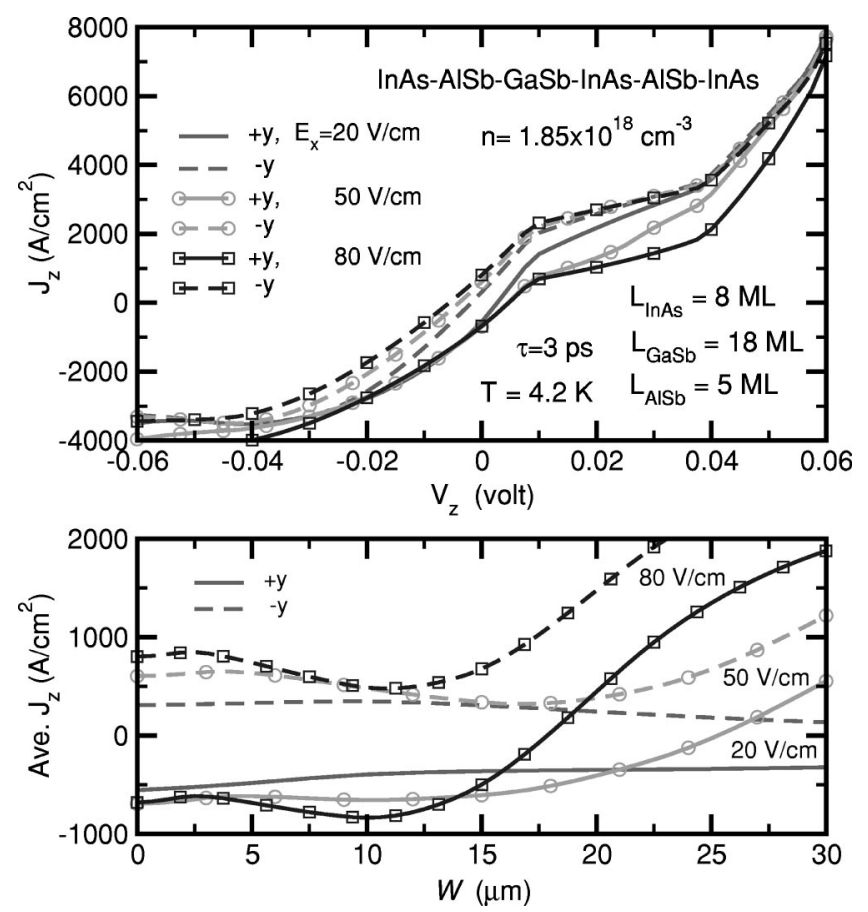

FIG. 6. Top panel shows spin polarized current-voltage characteristics of a spin pump structure at lateral $E$ field values of 20,50 (circles), and $80 \mathrm{~V} / \mathrm{cm}$ (squares). The bottom panel shows the averaged spin resolved current density components as functions of lateral device size $W$. lateral $E$ field. It shows that bidirectional spin pumping, i.e., oppositely spin polarized current components flowing in opposite directions, occurs only for a very limited range of vertical biasing values near $V_{z}=0$. However, the finite-size spin pump is aided by the following mechanism. Outside the small $V_{z}$ range where we have bidirectional flow, $J(+y)$ and $J(-y)$ flow in the same direction but can still differ substantially in size so that the aRITD behaves as a spin filter, favoring the transmission of one spin type over another. A pair of oppositely biased diodes, laterally located on opposite sides of the device, as illustrated in Fig. 5, could be considered as two complementary spin filters, sending oppositely polarized current components in opposite directions; together they again can act as a bidirectional spin pump. However, this mechanism can not be extended to arbitrarily large biases, since the difference between $J(+y)$ and $J(-y)$, hence, current spin polarization, decreases with increasing bias, rendering each of the paired spin filters themselves less effective. The bottom panel of Fig. 6 shows the averaged spindependent current densities in finite-size spin pumps as functions of lateral device size, computed using our parallel diodes model. For all three values of $E_{x}$, the device shows bidirectional spin pumping for lateral device size less than 15 $\mu \mathrm{m}$. Under larger $E_{x}$, the spin polarized current components tend to be somewhat larger, but smaller $E_{x}$ accommodates bidirectional spin pumping in wider devices.

The results shown in this work represent the initial response of the spin pump to the driving lateral $E$ field. The degree to which carriers in the electrodes are spin polarized in steady state depends on carrier densities, spin pumping efficiency, and spin relaxation processes. Spin-polarized currents should be detectable even if the carrier density spin polarization is small. ${ }^{11}$

The authors thank A. T. Hunter, D. L. Smith, D. H. Chow, J. S. Moon, and J. N. Schulman for helpful discussions. This work was sponsored by the DARPA under Contract no. MDA972-01-C-0002, and carried out in part at the Jet Propulsion Laboratory, California Institute of Technology, through an agreement with NASA.

${ }^{1}$ A. Voskoboynikov, S. S. Lin, C. P. Lee, and O. Tretyak, J. Appl. Phys. 87, 387 (2000).

${ }^{2}$ T. Koga, J. Nitta, H. Takayanagi, and S. Datta, Phys. Rev. Lett. 88, 126601 (2002).

${ }^{3}$ D. Z.-Y. Ting and X. Cartoixà, Appl. Phys. Lett. 81, 4198 (2002).

${ }^{4}$ Y. A. Bychkov and E. I. Rashba, J. Phys. C 17, 6039 (1984).

${ }^{5}$ A. Voskoboynikov, S. S. Liu and C. P. Lee, Phys. Rev. B 59, 12514 (1999).

${ }^{6}$ E. A. de Andrada e Silva and G. C. La Rocca, Phys. Rev. B 59, 15583 (1999).

${ }^{7}$ Our preliminary calculations show that although spin direction patterns are more complicated when anisotropy and bulk inversion asymmetry effects are included, basic conclusions on spin pumping remain valid.

${ }^{8}$ D. Z.-Y. Ting, E. T. Yu, and T. C. McGill, Phys. Rev. B 45, 3583 (1992).

${ }^{9}$ C. R. Bolognesi, J. E. Bryce, and D. H. Chow, Appl. Phys. Lett. 69, 3531 (1996); Yu. G. Sadofyev, A. Ramamoorthy, B. Naser, J. P. Bird, S. R. Johnson, and Y.-H. Zhang, ibid. 81, 1833 (2002).

${ }^{10}$ J. M. Ziman, Principles of the Theory of Solids, 2nd ed. (Cambridge University Press, Cambridge, 1972), pp. 215-219.

${ }^{11}$ J. D. Albrecht and D. L. Smith, Phys. Rev. B (in press) (cond-mat/0302457); D. L. Smith (private communication). 